Relations industrielles

Industrial Relations

\title{
Le tribunal du travail
}

\section{The Labour Court}

\section{André Rousseau}

Volume 25, numéro 2, 1970

URI : https://id.erudit.org/iderudit/028130ar

DOI : https://doi.org/10.7202/028130ar

Aller au sommaire du numéro

\section{Éditeur(s)}

Département des relations industrielles de l'Université Laval

\section{ISSN}

0034-379X (imprimé)

1703-8138 (numérique)

Découvrir la revue

\section{Citer cet article}

Rousseau, A. (1970). Le tribunal du travail. Relations industrielles / Industrial Relations, 25(2), 302-320. https://doi.org/10.7202/028130ar
Résumé de l'article

L'auteur étudie les dispositions nouvelles relatives au tribunal du travall dans le Code du travail du Québec.
Tous droits réservés (C) Département des relations industrielles de l'Université Laval, 1970
Ce document est protégé par la loi sur le droit d'auteur. L'utilisation des services d’Érudit (y compris la reproduction) est assujettie à sa politique d'utilisation que vous pouvez consulter en ligne.

https://apropos.erudit.org/fr/usagers/politique-dutilisation/ 


\title{
Le tribunal du travail
}

\author{
André Rousseau
}

L'auteur étudie les dispositions nouvelles relatives au tribunal du travail dans le Code du travail du Québec.

\section{Introduction}

Le projet d'un tribunal du travail n'est pas nouveau pour le milieu des relations de travail. Déjà, en 1940, Me Marie-Louis Beaulieu, à la demande du Ministère du Travail, préparait une «loi instituant des tribunaux du travail $»^{2}$. Le Congrès des relations industrielles de Laval, en 1954, portait sur le règlement des conflits de droit ${ }^{3}$ alors que le congrès de 1961 traitait spécifiquement de tribunaux du travail.

L'intérêt de la question n'est pas purement académique, car le Ministère du Travail a soumis en 1969 à la Législature des mesures dont l'effet est d'instituer un tribunal du travail et de modifier le Code du travail sur plusieurs points. Notre étude se limitera, à cet égard, aux dispositions nouvelles relatives au tribunal du travail. Le nouveau tribunal, créé aux termes de la loi modifiant le Code du travail (Bill 50),

ROUSSEAU, André, Département des relations industrielles, Université de Montréal.

1 Thèse de maîtrise soumise par l'auteur, en 1969 au département des relations industrielles de l'Université de Montréal.

2 Les Tribunaux du Travail: XVIe Congrès des relations industrielles (Laval) Les Presses de l'Université Laval, 1961. 162 pp. (p. 147 à 162).

3 Le Règlement des Conflits de Droit: IXe Congrès des relations Industrielles (Laval), Les Presses Universitaires de Laval, 1954, 132 pp. 
est institué pour avoir la compétence prévue au nouvel article 103 du Code du travail ${ }^{4}$.

«Ce tribunal a juridiction pour connaître et disposer, exclusivement à tout autre tribunal, en outre des autres matières qui sont déclarées par la loi être de sa compétence,

a) en appel, de toute décision d'un commissaire-enquêteur qui termine une affaire et de toute décision du commissaire-enquêteur en chef rendue en vertu de l'article 8 ou de l'article 9 ;

b) en première instance, de toute poursuite pénale intentée en vertu du présent code ».

Ce tribunal se présente donc comme le palier ultime du processus d'accréditation et comme une juridiction exclusive en matière d'infractions au Code du travail. Voilà, pour un nouveau tribunal, un cadre juridictionnel bien étroit, qui nous laisse songeur sur l'utilité de lui avoir donné le jour.

\section{État du droit et des recours}

Alors que se développe le droit statutaire en matière de travail, on continue de sentir l'influence du droit civil et de ses principes, qui s'appliquent, sauf dans la mesure ou des textes précis prévoient un régime spécial. Le contrat individuel de travail conserve sa vigueur et poursuit son existence parallèlement à la convention collective. Ce contrat obéit aux règles du droit commun : consentement libre, impossibilité de résiliation unilatérale, annulation pour cause de violence, fraude ou erreur. À cela s'ajoute tout le champ de la responsabilité pour dommages, rupture abusive de contrat, vices d'exécution ${ }^{5}$.

Il est vrai que les relations du travail échappent graduellement au droit commun : ainsi on ne saurait stipuler un salaire inférieur à celui que prévoit la Loi du Salaire Minimum, dans les milieux où cette loi s'applique. Mais il faut se garder de croire que le monde du travail soit complètement entré dans l'ère du collectif. Pour tous ceux qui oeuvrent

4 Loi modifiant le Code du travail (Bill 50) sanctionnée le 13 juin 1969, article 100 à 120 du Code.

Loi modifiant le Code du travail et d'autres dispositions législatives (Bill 65), article 28 modifiant à nouveau les articles 103 et 104 du Code du travail, adopté le 23 octobre 1969 et sanctionné le même jour.

5 Sur le contrat de travail, nous renvoyons à un article de Claude D'Aoust, auquel nous avons collaboré, à publier dans: Annotation et Jurisprudence des lois $d u$ Travail, Vol 2, F. Morin et J. Dupont, Québec, 1970. 
dans des secteurs marginaux, de même que pour les cadres non-salariés, l'organisation syndicale et la nouvelle législation ne sont d'aucune assistance; tous ceux-là, et ils représentent la majorité des travailleurs demeurent réduits à l'inefficacité des recours traditionnels devant les tribunaux.

Sous réserve du caractère réglementaire et impératif de la convention collective, le lien contractuel entre employeur et employé persiste, avec les droits et obligations qu'il comporte : prestation du travail, respect des directives patronales, paiement du salaire, avis de congé, au cas de terminaison d'emploi. En cas de litige sur le contenu de ces droits et devoirs, on en réfère aux tribunaux civils, qui jouissent d'une juridiction exclusive sur tous conflits dont le fondement est le contrat individuel, ce qui n'empêche pas les recours à la procédure de griefs, sous la convention.

La convention collective, par ailleurs, se présente comme un système autonome et complet jouissant d'une juridiction particulière, d'une procédure propre et de mécanismes caractéristiques. D'où elle connaît des recours spécifiques, qu'il importe de distinguer de ceux auxquels donne ouverture le droit commun.

Recours sous le droit commun et pénal

Il nous faudra nous borner à une énumération, car il n'entre pas dans le cadre de notre étude d'analyser tous ces recours. Ainsi, dans l'ordre du contrat individuel, on pense à des recours tels: l'action pour libérer un incapable de ses obligations; l'action en rescision et annulation de contrat aux motifs d'erreur, fraude ou violence; la rescision pour cause de lésion à l'endroit d'un mineur; la déclaration de nullité d'une obligation sans cause ou fondée sur une considération illicite; tous ces motifs constituent également des moyens de défense à des actions en exécution des contrats ${ }^{6}$.

Il faut aussi rattacher aux contrats la convention collective de droit commun, qui n'intervient pas en vertu du Code du Travail. Cette convention ne lie que les mandants des parties à la négociation et elle demeure facultative et particulière. L'exercice des recours nés de cette convention obéira aux normes du droit commun; par exemple, le demandeur devra justifier de son appartenance à un des groupes signataires. Les actions

6 D’une façon générale voir: Code Civil de la Province de Québec, Titre des Obligations, articles 984 à 1012 . 
dont les tribunaux sont saisis le plus fréquemment, en matière de travail, sont les poursuites en réclamation de salaire et en recouvrement d'indemnités, car les conventions collectives ne contiennent rien sur l'obligation de payer ce salaire; elles se résument généralement à établir l'échelle des salaires, qui sont dûs en vertu de la relation contractuelle entre l'employeur et l'employé 7 .

Les indemnités sujettes à recouvrement connaissent une grande variété: préavis de licenciement, prime d'éloignement, indemnité de déplacement, clause pénale garantissant l'exécution d'une obligation principale.

A moins que, par une clause pénale, on ait déterminé à l'avance les dommages exigibles dans le cas d'une exécution mauvaise ou tardive du contrat, c'est par une action en dommages-intérêts qu'il faudra se pourvoir. Outre l'action fondée sur contrat, il y a ouverture aux recours en responsabilité délictuelle, qui trouvent, en droit de travail, plusieurs manifestations ${ }^{8}$.

L'inventaire du recours sous le droit commun embrasse des mesures que le Code de procédure Civile qualifie de recours extraordinaires ${ }^{9}$. La Cour Supérieure, saisie de ces recours, se trouve constituée juge de la légalité des procédures ou des jugements des tribunaux inférieurs, de la légalité de l'occupation de charges publiques et de la légalité du refus d'accomplir un devoir que la loi impose.

Dans le cas des organismes habilités à entendre des litiges du travail, le respect de la légalité se traduit par le respect de la juridiction. Les moyens de soulever le défaut ou l'excès de juridiction sont les brefs de prohibition et l'action en nullité de la décision rendue; ce moyen de nullité peut également être opposé à une demande en exécution de la décision.

Outre la sanction de ce devoir public qu'est le respect de la juridiction, le droit commun permet un recours pour s'assurer de l'accomplis-

71967 R.C.S. 3 The Hamilton Street Railway Company c. Northcott Le juge Judson, à la page 5, déclarait: "The collective agreement is not concerned with nonpayment of wages. These may be sued for in the ordinary courts."

81967 B.R. 487 Gaspé Cooper Mines c. United Steelworkers of America (grève illégale, dommages aux biens et perte de production).

9 Code de Procédure Civile (Québec), "Titre Sixième, de Certains Recours Extraordinaires," articles 834 à 850 . 
sement des devoirs privés auxquels une personne a pu s'obliger: l'injonction ${ }^{10}$. Elle s'exerce soit de façon principale, sans autres conclusions, soit de façon interlocutaire, greffée à une action en dommages, par exemple.

Quant aux recours pénals qu'il nous suffise, à ce stade, de mentionner les matières de droit criminel susceptibles de trouver application en matière de travail: violation de contrat ${ }^{11}$; intimidation, menaces, violence et poursuite désordonnée ${ }^{12}$; méfait ${ }^{13}$; complot $^{14}$ et congédiement pour activités syndicales ${ }^{15}$.

\section{Recours statutaires}

Nous entendons par ce vocable tous les recours civils et pénals en vertu des diverses lois du travail. A titre d'illustration, mentionnons la plainte au tribunal du travail dans le cas d'ingérence d'un employeur dans l'association qui regroupe ses salariés ${ }^{16}$; un exemple de recours civil serait l'action qu'exerce un comité paritaire au nom des salariés visés par le décret ${ }^{17}$.

\section{Recours à l'arbitrage}

La convention collective, avons-nous dit, jouit d'une juridiction particulière; en effet, elle a pour tribunal le conseil d'arbitrage ou l'arbitre unique, selon le cas. Il est, sous le régime de la convention, trois types de conflit: le différend, le grief et la mésentente sur une matière non portée au texte de la loi des parties.

La solution par voie d'arbitrage est volontaire, dans le cas du différend, et obligatoire pour la solution des griefs. Quant à la mésentente pendant la période d'application de la convention, elle ne trouvera de solution pacifique et légale que si la convention prévoit un mécanisme de règlement, l'arbitrage par exemple. à 761 .

10 Code de Procédure Civile (Québec), "Livre Cinquième," ch. 3, art. 751

11 Code Criminel, 1953-1954, S.R.C. Ch. 51, art. 365.

12 Code Criminel, art. 366

13 Code Criminel, art. 372

14 Code Criminel, art. 410

15 Code Criminel, art. 367

16 Code du travail, art. 125, S.R.Q., Ch. 141.

17 Loi des décrets de convention collective, S.R.Q., Ch. 143, art. 20. 


\section{Avantages d'un tribunal du travail}

La création d'un tribunal du travail représente un apport plus ou moins positif au règlement des conflits de travail, selon le type même de l'institution retenue. Ainsi, nous croyons qu'il serait de peu d'utilité de substituer un tribunal au système d'arbitres de conflits de droit que nous connaissons actuellement, car nous pourrions difficilement imaginer un système plus accessible aux parties.

Au contraire, s'agit-il d'instaurer un tribunal du travail pour avoir une compétence embrassant tout le champ des conflits et des recours en matière de travail, alors nous soutenons que cet organisme unique, à caractère juridictionnel, justifierait une transformation des institutions que nous connaissons.

Organisme unique

L'organisme dont nous souhaitons la mise en place jouirait de la compétence exclusive pour décider des litiges suivants:

a) tous recours civils mettant en cause des personnes agissant en qualité de travailleurs ou d'employeurs, ou d'associations de travailleurs ou d'employeurs;

b) tous recours, civils et pénaux sous l'empire des lois administratives du travail;

c) toutes injonctions et tous recours extraordinaires exercés à l'encontre de personnes ou d'organismes chargés de l'application du droit de travail, ou à l'encontre de décisions rendues en la matière;

d) tous recours dévolus à l'actuel tribunal du travail;

e) tous recours à l'arbitrage des différends et des griefs.

Ainsi, la fonction juridictionnelle se trouverait réservée dans sa totalité à un tribunal du travail, sans pour autant priver les organes administratifs en place de leurs pouvoirs autres que judiciaire et quasi-judiciaire.

L'unicité de l'organisme répond à un double objectif: éliminer les conflits de juridiction et faciliter l'institution du recours. Qu'il nous suffise, sur le premier point, de signaler les conflits possibles entre les com- 
pétences dévolues au juge, à l'arbitre et parfois même à des organismes administratifs, qui se voient confier des pouvoirs quasi-judiciaires. À titre d'exemple, un congédiement en période d'organisation syndicale pourra donner lieu à trois recours, en vertu des lois provinciales : devant le tribunal ordinaire, au motif de rupture abusive de contrat, devant l'arbitre, au motif de renvoi sans cause juste et devant le commissaire-enquêteur en chef, en alléguant les activités syndicales. Ce dernier motif pourrait encore fonder un recours pénal, sous le code criminel.

Quant au second objectif, on conçoit aisément que le justiciable aura tôt fait de reconnaître l'organisme compétent, s’il est unique. Mais la facilité pour instituer le recours va plus loin: à cause de la multiplicité des organismes actuels, seuls des experts réussissent à s'y retrouver, car les règles varient selon le tribunal ou l'organisme et il faut rechercher dans un grand nombre de lois les règles attributives de compétence, et les normes régissant la procédure, la preuve et l'interprétation, sur lesquelles nous reviendrons un peu plus loin.

\section{Participation des intéressés}

Un voeu qui a été formulé, maintes fois depuis quelques années consiste en la reconnaissance du principe de la collégialité, dans l'adjudication des conflits. Ce principe cherche à rendre la justice plus consciente des problèmes du milieu qu'elle sert. Le moyen de mettre en oeuvre lla collégialité a été, pour la majorité des pays qui ont instauré le tribunal du travail, de faire appel à l'assessorat ${ }^{18}$.

Choisis au sein de listes établies d'avance par les intéressés et sans considération de conflits particuliers, les assesseurs représentent, auprès du juge du travail, les syndicats et le patronat. L'assesseur n'est pas que le représentant d'une partie, ni un simple expert, pour conseiller le juge. La majorité des législations pertinentes lui réservent un rôle actif, une participation à la décision finale.

Le rôle des assesseurs consiste d'abord à évaluer la force respective des parties et le but qu'elles poursuivent, pour en informer le président du tribunal; à titre d'experts, ils lui fournissent les données pertinentes sur

18 B.I.T.: La conciliation et l'arbitrage des conflits du travail, Études et Documents, série A, no 34, Genève, 1933 p. 48 et seq. Voir aussi la remise à jour de cette étude (pays d'Amérique) Études et Documents, nouvelle série no 13, Genève 1949. 
l'industrie en cause, sa situation économique, son système de relations de travail et tout ce qui peut contribuer à une juste appréciation du cas.

La présence d'un collège, formé du président et des assesseurs, contribue à faire participer au règlement des conflits des citoyens directement concernés, ce qui évite un divorce entre les conceptions sociales et économiques des magistrats professionnels et les conceptions des autres membres de la société. On met ainsi le tribunal à l'abri des reproches d'incompétence, de partialité ou de sclérose.

Solutions au niveau d'une industrie, d'un syndicat

La participation d'assesseurs bien informés des problèmes du milieu, d'une part, et l'étendue de la compétence accordée au tribunal, d'autre part, sont de nature à permettre et à favoriser la prise de décisions plus générales et l'atteinte de solutions plus globales. En effet, nous croyons qu'il serait désormais possible de saisir le tribunal du travail de l'ensemble d'un conflit, car l'unicité de l'organisme et sa vaste compétence éviteraient le morcellement des recours. Par ailleurs, l'assistance apportée au président par les assesseurs faciliterait l'ouverture du débat à sa dimension réelle, qui dépasse souvent le cadre d'une entreprise, d'un établissement ou d'un employeur particulier.

Les moyens actuels de solution sont trop partiels et trop occasionnels pour embrasser des solutions d'ensemble aux problèmes du travail. L'inventaire des recours aura manifesté le grand nombre d'organismes compétents et la confusion qui existe dans le choix des recours appropriés. Or, aucun des mécanismes en place ne jouit d'une autorité assez vaste et suffisamment forte pour répondre adéquatement aux problèmes globaux qui se posent à l'occasion de conflits en apparence particularisés.

C'est précisément le système de règlement de conflits qui impose aux intéressés de scinder et diviser les problèmes, pour les présenter aux multiples juridictions habiles à les entendre. Pour assurer au tribunal l'aptitude à envisager les impératifs supérieurs d'une industrie, d'un secteur, ou d'une organisation syndicale, le choix des assesseurs pourrait se faire sur une double base: par référence à un secteur industriel, une profession ou un service, et sur une base géographique.

On comprend que le président du tribunal ne puisse se familiariser avec les données économiques, sociologiques et politiques d'une foule 
d'entreprises et d'industries, et qu'il ne puisse connaître à fond les diverses associations syndicales; c'est là qu'interviennent les assesseurs, pour l'informer de ces considérations économiques et autres, lui faire part du type de relations de travail dans telle industrie, et lui faire connaître l'évolution de ces mêmes relations.

En possession de ces données, le président et les assesseurs sont plus en mesure d'élaborer une solution qui tienne compte des besoins non seulement immédiats, mais encore des exigences plus lointaines du milieu où a germé le conflit.

En cette matière, il nous faut déplorer l'approche traditionnelle qui, veut éviter la généralisation des conflits et qui tire de cet objectif valable, un prétexte pour n'édicter que des solutions partielles aux conflits qui se présentent. Nos lois du travail et la réglementation qui en résulte constituent des compromis entre différents secteurs de la société. De la même façon, la convention collective réunit dans un pacte à durée limitée des groupes dont les intérêts s'opposent et divergent souvent. La difficulté de réaliser ces compromis accroît d'autant l'importance des mécanismes auxquels on confie leur interprétation et leur mise en oeuvre.

Or c'est bien souvent par le biais de griefs individuels et particuliers que se trouve contesté le mérite du compromis auquel on est arrivé. En effet, ce sont des intérêts individuels qui se trouvent représentés devant les cours civiles et les arbitres. Comme le déclarait Me Émile Gosselin: 19

«Mais en matière de droit du travail, nombreuses sont les décisions qui s'appliquent directement aux individus, mais qui sont de nature à modifier l'équilibre social, donc à changer la portée du compromis entre de vastes secteurs de la société ».

Au delà du cas d'espèce soumis à l'organisme juridictionnel, en vue de régler le problème d'un individu ou d'un groupe particulier, il y a une décision qui affecte le compromis social réalisé par les parties ou par un organisme administratif; si bien que toute la relation de travail peut se trouver mise en cause à l'occasion du cas sous étude. Cette relation de travail se présentant bien différemment selon le type d'employeur, l'industrie ou le mode d'organisation et de représentation syndicale, il nous semble important que toute décision affectant cette relation soit prise en connaissance des éléments constitutifs du milieu où se vit la relation de travail.

19 Gosselın, Émile: Nature et raisons d'être des tribunaux du travail. dans: Les Tribunaux du Travail, cité en note (2) - (p. 32) 
Cette connaissance manque hélas! trop souvent aux juges et arbitres appelés à se prononcer sur des conflits de travail. Les tribunaux civils, en particulier, demeurent des organismes essentiellement préoccupés de rapports entre individus; de ce fait, il leur est très difficile et même impossible, dans le cadre des lois qu'il leur incombe d'appliquer, de dépasser la spécificité d'un conflit pour envisager des voies de règlement collectives, ou orientées sur des entités qui échappent au niveau immédiat qui a vu éclater le conflit, né ailleurs bien souvent.

Règles d'interprétation, de preuve et de procédure

$\mathrm{Si}$ nous connaissons relativement bien les règles suivies par les cours en matière d'interprétation, de preuve et de procédure, nous pouvons plus difficilement identifier les règles qui guident, en ces matières, les conseils d'arbitrage des différends et les arbitres des griefs. Quant à la CRT un ex-président a déjà rappelé qu'elle obéissait aux règles de preuve et de procédure dictées au Code Civil et Code de Procédure ${ }^{20}$.

Dans le cas des conseils d'arbitrage, ils ont autorité sur la preuve et la procédure ${ }^{21}$ si bien que la conduite de l'enquête n'y obéit qu'aux normes fondamentales de justice et aux prescriptions du conseil. Quant à l'arbitre des griefs, le Code du travail ne nous aide pas à préciser les règles qui le guideront dans l'interprétation des conventions collectives, non plus que la procédure et le mode de preuve qu'il devra retenir. Comme sa compétence se fonde sur la convention, celle-ci pourra établir ces règles, mais en pratique peu ou pas de conventions ne l'ont fait.

Aussi nous trouvons-nous dans la situation de normes qui fluctuent, si bien que la conduite des enquêtes n'a rien de bien arrêté. À ce point de vue, un organisme unique pour adjuger des conflits du travail aurait tôt fait d'édicter ou de se voir imposer des règles uniformes pour l'interprétation des textes et la conduite de ses enquêtes. À court terme, nous croyons que l'on pourrait importer les règles que connait le droit civil en ces matières, car elles ont subi l'épreuve du temps et s'avèrent utiles pour la protection du justiciable. À long terme, nous suggérons de ne retenir que les principes d'interprétation et d'assouplir les exigences de preuve et de procédure devant le tribunal du travail, à cause du caractère d'urgence des conflits du travail.

201967 R.D.T. 222 (p. 234). Regards sur l'évolution jurisprudentielle et la C.R.T. (Juge A.B. Gold)

21 Code du Travail, article 69. 
Accessibilité à tous les travailleurs

Le syndicalisme et la négociation collective touchent encore moins de la moitié de la main-d'oeuvre non agricole rémunérée ${ }^{22}$. Mème si la liberté d'association et le pouvoir de négociation ont fait des gains intéressants, cet élargissement n'a pas apporté de réponse au groupe imposant de travailleurs qui oeuvrent dans des secteurs économiques marginaux et pour qui l'organisation syndicale n'est guère possible.

Ainsi, des groupes comme les professions traditionnelles, les travailleurs agricoles et les domestiques se trouvent exclus par la législation. Beaucoup d'autres travailleurs sont exclus à cause de la difficulté de les regrouper à l'intérieur d'unités de négociation telles qu'elles existent actuellement. La loi exclut encore le personnel de direction. Pour tous ces gens à qui on refuse le bénéfice de la syndicalisation ou qui s'en trouvent privés, faute d'un milieu propice à l'organisation, il n'est de recours que devant les tribunaux ordinaires, pour assurer la protection de leurs droits.

Pour beaucoup d'entre eux, ces groupes se trouvent déjà dans une situation de marchandage défavorable et ils parviennent à grand peine à défendre leurs intérêts au moment de la négociation des conditions de travail. Il leur sera tout aussi onéreux, et parfois impossible de veiller au respect de leurs droits, à cause des délais et des coûts prohibitifs que signifieraient des procédures judiciaires.

La compétence très vaste que nous avons suggérée de reconnaître au tribunal du travail nous apparaît un moyen de pallier à ces dénis d'action, à toutes fins pratiques. La souplesse de la procédure et des modes de preuve, la suppression des moyens dilatoires, l'accessibilité du tribunal, voilà autant de moyens d'assurer l'exercice des recours auxquels trop de travailleurs renoncent dans l'état actuel du droit, car ils ne peuvent s'en payer le luxe.

\section{Objections à l'encontre de l'institution d'un tribunal du travail}

Nous sommes conscients que la création d'un tribunal du travail ne va pas sans quelques problèmes. Sans nous arrêter aux objections d'ordre constitutionnel, car cette question a déjà fait l'objet de quelques excellents

22 Les Relations du Travail au Canada, Rapport de l'Équipe spécialisée en relations de travail, Ottawa 1968, 272 p. (page 95). 
articles ${ }^{23}$, nous signalons un triple obstacle constitutionnel: quels tribunaux une province peut-elle valablement constituer et de quelle juridiction peut-elle les investir? Comment peut-on soustraire un tel tribunal au pouvoir de revision et contrôle de la Cour Supérieure? Peut-on recruter la magistrature en dehors des cadres du Barreau?

Il semble que des solutions intéressantes à ces questions commanderaient des modifications au texte constitutionnel. Sur les modifications nécessaires, ou les moyens d'y obvier, nous renvoyons le lecteur aux articles cités.

Structures économiques et institutionnelles axées sur l'entreprise

Notre système économique, fondé sur le libre marché et le libre jeu de l'offre et de la demande, s'inscrit dans une philosophie plus générale de libéralisme. D'où les éléments déterminants du système sont, quant à la situation du travailleur, la liberté des conventions et la liberté de commerce.

Ces libertés, à cause du déséquilibre des forces en présence, ont souvent joué à l'encontre du travailleur individuel; la reconnaissance du droit d'association et de coalition a favorisé l'organisation syndicale et a contribué au développement de la négociation collective, mais cet effet n'a pas été senti dans tous les secteurs de la vie économique.

Comme nous le rappelions plus haut, une grande partie des travailleurs ne peut encore compter dans l'élaboration des conditions de travail, que sur les moyens de la négociation individuelle avec l'employeur. Il en va autrement dans les milieux où a pénétré l'organisation syndicale; là, les conventions collectives jouent un rôle important dans la fixation des conditions de travail.

La fixation des règles et normes qui présideront à la relation de travail, par la mise en vigueur de conventions collectives, demeure essentiellement, chez nous, un phénomène axé sur l'entreprise. C'est avec un

23 Yaccarini, Benoît. "Les Tribunaux du Travail en général et dans la Province de Québec," 9, Revue du Barreau, 1949, p. 379-446.

LaPoINTE, Marc. "La place des tribunaux du travail dans l'ensemble de l'organisation judiciaire," dans: Les Tribunaux du Travail, Presses de l'Université Laval, 1961, p. 101 à 106. 
employeur, une entreprise ou un établissement donné que le syndicat négocie; le syndicat se définit, sur le plan de l'accréditation, par son rattachement à un employeur ou une entreprise.

Une conséquence inhérente et peut-être nécessaire du régime de négociation collective au niveau de l'entreprise, nous apparaît être le caractère privé du système de règlement de grief et d'arbitrage des conflits. Sans préjuger pour l'avenir du voeu des intéressés, on peut les croire plus soucieux de se donner eux-mêmes les mécanismes dont ils ont besoin, qu'enclins à favoriser la mise en place de tribunaux publics.

Le type ou le modèle de relations de travail que nous connaissons n'est pas étranger au fait que bon nombre d'entreprises et de syndicats représentent des institutions qui dépassent notre frontière, et s'inspirent du modèle américain, qui est le leur. Qu'il nous suffise de rappeler la réticence avec laquelle les partenaires américains à la négociation font appel à l'intervention étatique. En tout état de cause, on cherche à conserver le mécanisme de règlement de conflits aussi près que possible de la source de ces conflits et des parties qui ont créé le mécanisme.

Aussi, un des arguments majeurs à l'encontre d'un tribunal du travail vient-il de l'esprit même avec lequel les intéressés entendent régler leurs conflits. Dans les milieux qui bénéficient des lois du travail, on entend peu de voix s'élever pour réclamer l'institution d'une juridiction du travail. Quant aux travailleurs non syndiqués, leur isolement les empêche sans doute de se faire entendre.

\section{Absence de ressources humaines}

La question d'un personnel suffisant et compétent ne se résout certes pas par des voeux pieux. Où trouver les juristes, économistes, spécialistes en relations de travail et tous autres experts que nécessitera la mise en oeuvre d'un tribunal du travail? Pour recevoir un bon accueil, le tribunal doit donner des garanties de viabilité telles que les parties intéressées aideront l'organisme à faire ses premiers pas.

Il faut encore envisager que l'éventuelle mise sur pied d'une équipe de recherche auprès de ce tribunal, comme l'a annoncé le ministère du travail et de la main-d'oeuvre, nécessitera le recrutement d'experts, qui sont denrée relativement rare. 
Pour le choix des assesseurs, nous croyons que le patronat et les syndicats sauraient dénicher, au sein de tous les services et industries majeurs de notre société, une pléiade de gens capables d'instruire les présidents du tribunal et habiles à siéger avec eux. Déjà, lors des conflits d'intérêts, on procède à la formation de conseils d'arbitrage à trois, où tous participent à la décision.

Le recrutement des présidents du tribunal soulève un problème plus sérieux. Comme nous proposons de reconnaître au tribunal une compétence pour le règlement des différends, il faudrait y désigner des présidents non-juristes, capables d'apprécier les questions économiques et techniques de la négociation.

Pour pallier au problème du manque de personnel, nous pensons à une formule de tribunal itinérant, avec présence de greffiers dans tous les centres qui justifieraient, par leur activité économique ou leur population, une telle présence. En conférant aux juges une juridiction à l'échelle de la province, on pourrait constituer, à Montréal et à Québec, des groupes plus nombreux qui pourraient, au besoin, assister le juge d'une certaine région ou s'y suppléer. Cette solution serait particulièrement indiquée pour le règlement des conflits d'intérêts, car le juge en chef pourrait alors déléguer un président particulièrement apte en cette matière.

Le problème du capital humain demeure car, actuellement, la grande majorité de ceux qui oeuvrent dans les relations de travail et qui siègent comme arbitres de conflits, le font parallèlement à l'exercice d'une autre profession, où ils sont en contact avec le monde du travail. Il n'est pas certain que beaucoup d'entre eux pourraient ou voudraient se libérer de ces occupations pour assumer des postes au tribunal du travail.

Organisme statutaire, imposé aux parties

Cette question rejoint celle des structures institutionnelles, abordée plus haut. Compte tenu de la volonté des parties de se doter elles-mêmes des mécanismes nécessaires, quel accueil réservera-t-on à une institution venue d'en haut, imposée par l'autorité gouvernementale? Patrons et syndicats n'y verraient-ils pas une intervention indue dans la vie des relations de travail?

Il incombe à l'État, bien sûr, de rechercher un système toujours plus cohérent dans les relations de travail et de favoriser la paix industrielle. 
Dans la poursuite de ces fins, l'État se trouve appelé à légiférer de façon accrue, en vue de rationaliser et d'intégrer les divers mécanismes de règlement de conflits.

Il ne faut pas oublier qu'il n'y a pas que les milieux syndiqués qui soient en cause. Le tribunal du travail nous apparaît d'abord et surtout une réponse aux problèmes des travailleurs non organisés, qui ont de sérieux griefs à l'encontre de l'administration judiciaire, car elle leur est quasi inaccessible.

En dépit des avantages que nous avons attribués à la création d'un tribunal du travail, il n'est pas sûr que les parties voudraient renoncer aux modes actuels de règlement. Il est plausible qu'on préfère ramener les conflits en termes d'une entreprise donnée et d'un syndicat particulier, et il y a de bonnes raisons de vouloir conserver ce modèle, car les parties s'assurent le contrôle des mécanismes de règlement.

\section{Absence d'une législation unifiée}

A un droit autonome et particulier doit répondre un ordre juridictionnel spécialisé. Mais encore faut-il se demander avant d'instaurer un tribunal spécialisé, s'il existe vraiment un ordre juridique autonome. Pouvons-nous identifier aisément, dans notre droit, les règles propres au domaine du travail?

Les textes législatifs relatifs aux questions du travail sont très dispersés, tant dans la législation fédérale que provinciale. Il est vrai que, dans un droit dont les limites ne sont pas encore fixées, une codification serait continuellement à refaire. D'où, il nous faut nous résigner à une législation sporadique.

L'unité du droit du travail sera longue à faire pour une autre raison: notre droit du travail, tout en entrant dans l'ère du collectif, conservera encore longtemps des amarres le rattachant au droit privé. Jusqu'à un certain point, il serait dangereux qu'il en fût autrement, car il faut souvent faire appel aux règles du droit commun pour suppléer aux carences de la législation du travail.

Ainsi le caractère disparate et incomplet du droit du travail constitue-til une réserve majeure à la création de tribunaux spécialisés. La mise en 
place soudaine d'une nouvelle institution ne changerait rien aux règles juridiques qui régissent les rapports de travail des gens non visés par les lois statutaires du travail. Pour ceux-là, le droit commun continuerait de s'appliquer et la jurisprudence seule ne suffirait pas à modifier la situation de ces travailleurs.

Faible concentration industrielle et accessibilité de l'organisme

L'accessibilité s'entend de la facilité pour le justiciable de repérer l'organisme compétent à entendre le problème qu'il veut lui soumettre; elle embrasse aussi la question du coût de la procédure et de la facilité d'accès au lieu où se déroulera le débat.

Pour le salarié lésé dans ses droits, les recours judiciaires civil ou pénal ne présentent pas une solution adéquate, car ils signifient un investissement, des frais, des déplacements et une perte de travail, s'il doit se présenter à l'audience en un lieu éloigné de son poste de travail. La multiplicité des recours conduit l'intéressé à ne plus savoir s'il doit agir seul, conjointement avec un organisme, avec ou sans autorisation administrative, ou si l'organisme prendra action pour lui.

Le regroupement de tous les mécanismes en une juridiction unique solutionnerait-il ces problèmes? Compte tenu du peu de ressources humaines et de la faible concentration industrielle, comment rendre le tribunal du travail accessible à tous les centres industriels importants?

On peut imaginer un assouplissement de la procédure, mais dans le cas des travailleurs syndiqués, on ne saurait rendre le tribunal plus accessible et moins coûteux que les mécanismes actuels d'arbitrage. Quant aux travailleurs réduits aux actions devant les tribunaux ordinaires, leur situation pourrait certes s'améliorer, dans l'hypothèse où l'on serait en mesure d'assurer la présence du tribunal du travail en maints endroits dans la province.

Aussi avons-nous retenu la formule de tribunaux itinérants, capables de siéger régulièrement dans un certain nombre de centres urbains. Nous n'avons pas comme projet de décrire tout le mécanisme qu'on pourrait mettre en oeuvre, mais nous croyons qu'il serait aisé d'édicter une procédure souple, analogue à celle que connaissent nos conseils d'arbitrage. 


\section{Conclusion}

Dans une étude sur les conseils de Prud'hommes en France, McPherson et Meyers rappellent les considérations principales qui ont favorisé la création de tribunaux du travail dans les pays industrialisés de type capitaliste: ${ }^{24}$ assurer la solution des conflits par des personnes compétentes, réaliser un effort maximal en vue de la conciliation, obtenir un règlement plus rapide que devant les tribunaux ordinaires, permettre une procédure plus souple et moins légaliste et réduire les frais pour le justiciable.

Nous avons voulu nous arrêter à des considérations plus particulières à notre milieu, ayant à l'esprit que les motifs et avantages énoncés plus haut jouaient en faveur de la création d'un tribunal chez nous, comme ailleurs, avec une réserve, savoir l'effort de conciliation. En effet, au moins dans les milieux régis par une convention collective, la conciliation a lieu, chez nous, au stade de la procédure de grief.

À cause de la compétence que nous suggérons de reconnaître au tribunal en matière de différends, nous croyons devoir souligner que fort peu de législations ont retenu cette solution. La majeure partie des pays distinguent entre les questions sur lesquelles un tribunal peut adjuger, et celles qui relèvent de la réglementation et de la négociation.

Dans une étude du tribunal du travail suédois, Robbins rappelle cette compétence « judiciaire » du tribunal comme un facteur important du succès que cet organisme a connu ${ }^{25}$. Notre argument principal, sur cette question, est le souci de créer un organisme unique, car nous sommes d'avis que le meilleur juge du conflit d'interprétation d'une convention collective serait ce juge qui a connu les difficultés de réglementation et de négociation à l'origine.

Nos institutions actuelles nous obligent à scinder les problèmes et à en particulariser l'approche. Or, il est indéniable que l'adjudication sur un point litigieux de convention collective ou de contrat de travail aura beaucoup plus de chances d'être satisfaisante si elle est confiée à l'orga-

24 McPherson, William H. et MeYers, Frederic. The French Labour Courts: Judgment by Peers, University of Illinois, 1966, pp. 104 (page 67).

25 Robins, James J. The Government of Labor Relations in Sweeden, Chapel Hill, New York, 1942, 367 pages, Ch. 11: "The Labor Court", Ch. 12: "Jurisdictional Boundaries", p. 187 à 232. 
nisme qui connaît bien le type de relations existant entre les parties, les difficultés qu'elles ont rencontrées à l'étape de la négociation et, d'une façon générale, qui connaît les parties mêmes à ce conflit.

En rapprochant ainsi la source législative et l'interprète de la loi, on assure, croyons-nous, une interprétation beaucoup plus respectueuse de l'intention des parties qui ont légiféré. Ce faisant, on accroît les garanties de paix industrielle, car les solutions aux conflits seront mieux adaptées et plus réalistes, tout en demeurant susceptibles d'envisager les problèmes globalement, au niveau d'une industrie, d'un secteur ou d'une centrale syndicale.

\section{THE LABOUR COURT}

The Province of Quebec has launched a major experiment by creating a Labour Court, constituted by Bill 50 (as revised by Bill 65). The Court's jurisdiction was defined by these two measures and is set out in Section 103 of the Quebec Labour Code.

The whole area of labour relations is marked increasingly by a collective approach, emphasizing collective bargaining. On the other hand, the Civil Code still prevails and civil courts remain the main recourse for non-unionized workers. The latter cannot take advantage of the new legislation, which protects only those who are in, or covered by labour organizations.

Even in union-organized sectors, we still note the necessity to maintain some recourse to common law tribunals; such recourse, dealing with the individual contract of employment and violations of criminal and statutory laws, is the only one available to workers in marginal and unorganized sectors, who still make up the majority of our labour force.

This paper proposes the creation of a Labour Court that would replace all the present dispute settlement procedures. This body would be competent to hear and decide : civil action between workers and employers; civil and criminal cases under statutory legislation in labour matters; injunctions and special writs in the field of labour law; action now before the labour Court; arbitration of disputes and grievances.

A Labour Court, under this very broad concept, would offer the following advantages: single and integrated jurisdiction, intervention by the interested parties, settlement at a higher level, uniform, rules of interpretation, of evidence and procedure, and easier access to all workers anxious to use its facilities.

We realize that such a proposal and such a transformation in our institutional structure would not be easy. The main objections to be encountered would appear to be : the fact that our economic and institutional structures are oriented towards the entreprise; the need for human resources, in order to provide all the specialists 
required by such a Labour Court; the statuory character of the Court, as against a contractual type of machinery which might be preferred by the parties; the lack, at the present time, of a unified body of laws, in the field of labour relations; the low industrial concentration, inview of the number of Courts to be created.

In conclusion, the principal advantage stressed is the possibility, through such a Labour Court, of changing the scope and level of decision-making. At the present time, the approach to labour problems is very specific and localized; the presence of the parties and the vast jurisdiction of a Labour Court would make it possible to take into consideration the problems of an entire industry, union or economic sector.

\section{SERVICE D'INFORMATION SUR LES CONVENTIONS COLLECTIVES}

Négociateurs patronaux et syndicaux qui avez besoin d'un supplément d'information sur

les conditions de travail

les salaires

les avantages sociaux

les changements technologiques

la sécurité d'emploi, etc. prévalant dans votre localité, région ou secteur industriel LE SERVICE D'INFORMATION SUR LES CONVENTIONS COLLECTIVES, Département des Relations industrielles, Université Laval, peut apporter une réponse à vos questions

- Qui peut utiliser le service?

tout organisme intéressé par la négociation de conventions collectives de travail.

- Quel genre de renseignements offre le service?

chaque projet d'analyse de contenu est conçu de façon à répondre aux besoins particuliers de chaque demandeur.

- Quel est le coût d'un tel service?

tout projet est évalué en fonction du travail qu'il exige et fait l'objet d'un contrat particulier.

- Où s'adresser?

\section{SERVICE D'INFORMATION SUR LES CONVENTIONS COLLECTIVES DÉPARTEMENT DES RELATIONS INDUSTRIELLES}

Université Laval, Québec 10, P. Q. 